\title{
DEFORMATION MODES AND STRUCTURAL RESPONSE OF DIATOM FRUSTULES
}

\section{ALEJANDRO GUTIÉRREZ ${ }^{1}$, RICHARD GORDON² and LILIAN P. DÁVILA ${ }^{1}$}

${ }^{1}$ Materials Science and Engineering

School of Engineering

University of California at Merced

5200 N. Lake Road

Merced, CA 95343

USA

e-mail: ldavila@ucmerced.edu

${ }^{2}$ Embryogenesis Center

Gulf Specimen Aquarium and Marine Laboratory

222 Clark Drive Panacea

FL 32346

USA

\begin{abstract}
The ubiquitous diatoms, single-celled algae with porous silica frustule (shell) morphology and features spanning multiple length scales, offer a promising foundation to convert knowledge of biological structures into novel design techniques. Diatom frustules are often found in nature with distinct deformation patterns suggesting the involvement of mechanical interactions in their morphogenesis. Understanding this phenomenon can lead to potential manufacturing techniques based on the micromanipulation of bio-inspired structures. In this study, the mechanics of centric diatom frustules was investigated
\end{abstract}

Keywords and phrases: diatoms, frustule deformation, microscale, nanoscale, biostructures, porous silica.

Received March 26, 2017; Revised April 28, 2017

(C) 2017 Scientific Advances Publishers 
using the finite element method (FEM) in combination with morphology and material properties obtained from scanning electron microscopy (SEM) and mechanical tests respectively. SEM micrographs of a marine diatom, Coscinodiscus sp., have allowed the classification of specific deformation patterns frequently observed on these frustules. To elucidate the nature of these deformations, a computer aided design (CAD) model approximating a centric diatom frustule was created. Using shear-deformable frustule finite elements and diatom-specific Young's modulus, critical buckling load and modal analysis of the diatom model were performed to investigate possible correlations of the deformation modes with observed morphologies. Results reveal that the first ten deformation modes correlate noticeably well with deformation patterns observed through SEM. Additionally, FEM models were used to study the relation between diatom morphology and mechanical behaviour. A quadratic relation was established between frustule pore size and critical buckling load as well as a cubic relation between frustule thickness and critical buckling load, reported for the first time in this study. The findings in this research provide insights into the mechanical response of diatom frustules that can aid the realization of tailored properties in new bio-inspired materials, in particular for nanotechnology applications, but also for advanced metamaterials and optomechanical devices.

\section{Introduction}

One of the grand challenges of science [1] is the manipulation of materials at the nanometer scale to obtain tailored properties. The achievement of a robust and practical method for such manipulation would drastically advance novel design and manufacturing techniques so that they would be no longer limited by the properties of the raw materials used, but instead by the smallest scale at which materials can be manipulated [2]. Toward achieving this ambitious goal, an effective path forward is the study of biomaterials to elucidate methods by which nanostructures found in nature can be adapted into human-made devices $[3,4]$. Organisms often develop biosilica structures with a level of complexity that surpasses their geological and synthetic counterparts [5]. Silica biostructures, produced by diatoms or radiolarians, have intricate morphologies tailored by evolution that appear to optimize specific functions such as protection from predators or filtering [6]. 
This study focuses particularly on diatoms, an important group of microscopic algae found in almost every aqueous environment on our planet [7]. There are more than 200,000 species of diatoms estimated to date [8] with a rich variety of size and morphology. Their extraordinary diversity and complex architecture rich in nanoscaled pores and slits represent a clear opportunity for investigators seeking sources of inspiration for innovative micro and nanodevices. Indeed, the unique structure of the diatom frustule has motivated researchers to propose potential applications in nanodevices [9], photonics [10], molecular separation and microfluidics [11], and drug delivery [12] among others. Additionally, the frustule architecture of diatoms carries considerable interest in itself, as these silica structures are both strong and lightweight. Diatoms have also been increasingly used as inspiration for the design of civil structures [13] and mechanical components [14].

Diatoms have a basic structure of porous silica layers in a hierarchical arrangement constituting a single valve [7]. A diatom frustule consists of two overlapping valves joined by one or more girdle bands, giving the frustule a resemblance to a Petri dish. Diatom frustules can vary in size from a few microns to over a millimeter [9] and they exhibit features (e.g., pores, slits, ribs, knobs) that can be as small as five nanometers [15]. Their multiscalar silica structure thus spans over eight orders of magnitude. There are two general types of diatoms depending on symmetry [16]: centric (with radial symmetry, often circular) and pennate (elongated and usually with bilateral symmetry). Examples of both types of diatoms are shown in Figure 1. 

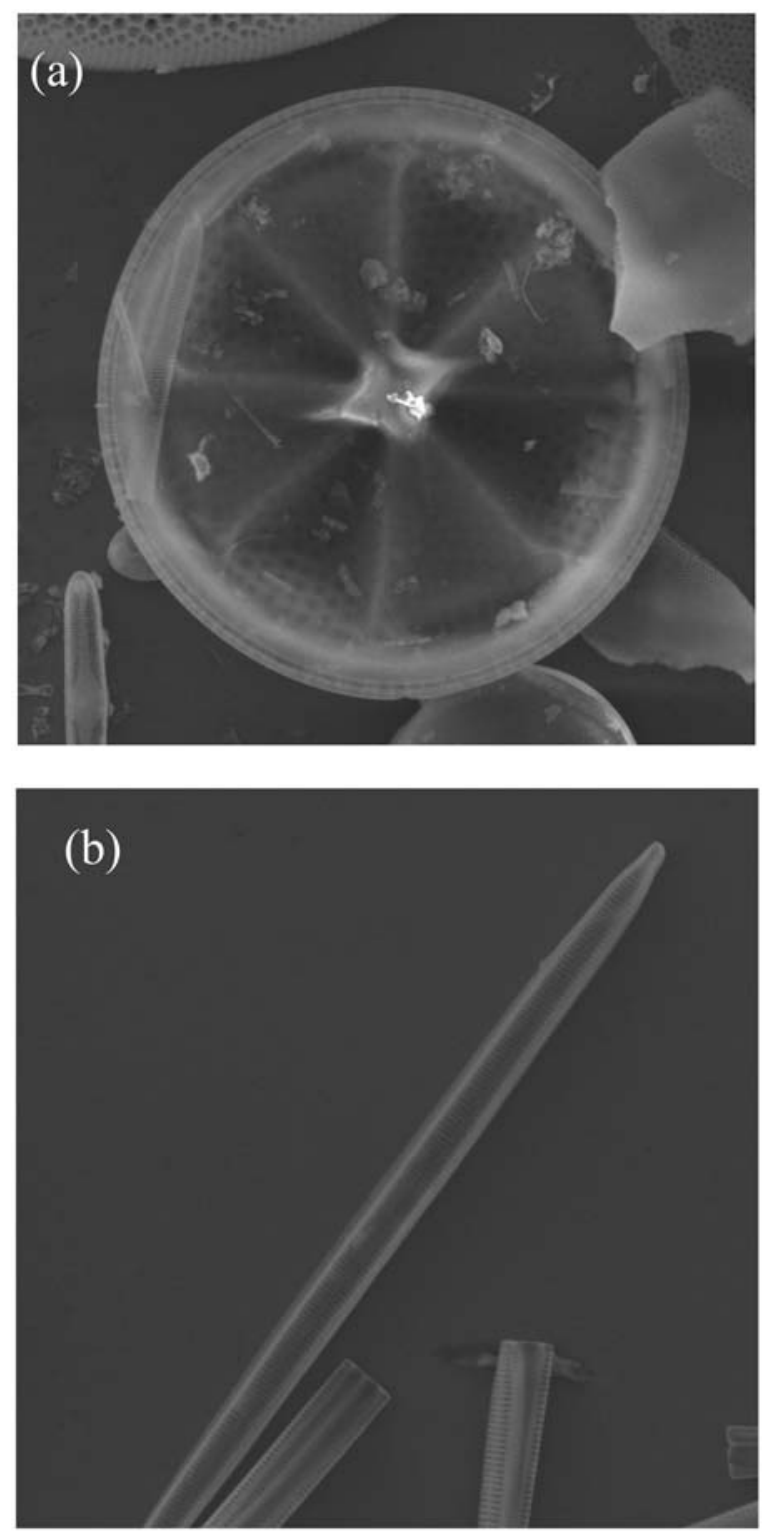

Figure 1. SEM images of typical diatom frustules (single valves) showing their distinct morphology: (a) centric Actinoptychus and (b) pennate Navicula. No observable deformation patterns or buckled features are detected in either diatom frustule. 
The mechanical behaviour of diatom frustules has been studied using micro- and nanomechanical testing as well as atomic force microscopy (AFM), scanning electron microscopy (SEM), and the finite element method (FEM). Hamm et al. [6] investigated the potential of diatom frustules as armor against predators based on microneedle loading tests, discovering an inverse relation between frustule size and mechanical strength up to the point of fracture. The investigators further elaborated [17] on the relation between the structural components of the frustule and the mechanical loads that diatoms experience in their natural environment using both experiments and simulations. Almqvist et al. [18] used AFM to measure elasticity and hardness in the frustules of the pennate diatom species Navicula pelliculosa and found the values of Young's modulus $E$ varied from the tens to the hundreds of $\mathrm{GPa}$ depending on the specific point over the frustule on which the tests were performed. The authors also reported a similar situation for hardness values, $H$ varying from 1 to $12 \mathrm{GPa}$. In the same way, Subhash et al. [19] reported notoriously localized behaviour over the frustules of Coscinodiscus sp., including differences in the failure mode when the frustules were loaded in the concave or convex orientations. Later, Losic et al. [20] used SEM to characterize the morphology of Coscinodiscus $s p$. and performed AFM nanoindentation tests on the frustules, finding significant variations of the elastic modulus $E(1.7-15.6 \mathrm{GPa})$ and hardness $H$ (0.076-0.53GPa) measurements depending on frustule morphology and on the location of the indentation, pointing towards highly localized properties. These results suggest that mechanical properties may be heavily dependent on morphological features. Since the complexity, variety, and size range of said features is vast, predicting the mechanics of diatom-inspired structures is far from a trivial pursuit. A useful research technique that reduces cost and time (i.e., due to the cumbersome and expensive battery of tests required for the mechanical study of diatom frustules) is numerical simulation using FEM, which is at the basis of this study. 
The use of mechanical simulation to study biomaterials has been steadily gaining momentum in recent years. One feasible approach is simulation based on atomistic principles, such as that reported in the work by Buehler and Gao [21], who used molecular dynamics (MD) to demonstrate the principle of flaw tolerance by size reduction in bone-like materials as well as the adhesion capabilities of geckos and insects. A similar approach was followed by Keten et al. [22] to study the effect of nanofeatures on the mechanical toughness of silk, contributing to explaining how size effects can be exploited to dramatically increase the mechanical properties of biomaterials. In the specific case of diatoms, the limited number of mechanical simulation studies emphasizes the need to evaluate the mechanical response of frustules at the microscale. In earlier work by Garcia et al. [23], MD simulations of diatom-inspired nanoporous materials were performed to investigate the effect of hierarchical nanoporous layers under tensile deformation. Although such simulations reported interesting material design insights at the atomicscale, the MD method is known to be computationally expensive for large and complex models. An alternative method is reported in a study by Hamm and co-workers [6] who employed FEM simulation of a Fragilariopsis kerguelensis pennate frustule to evaluate its strength in the event of a predator attack. Using hexahedral elements, the frustule was simulated under uniform external pressure and patch loading to simulate a mandible bite. Results from that study showed a significant influence of the morphology and the nanofeatures (in this case, ribs) on the resistance of the diatom frustule to these attack events. More recently, the work by $\mathrm{Lu}$ et al. [24] focused on the frustule of Coscinodiscus sp. as a cellular structure and modelled the frustule as a hexagonal multilayer mechanical component under a uniform compression. The stress distributions throughout the porous frustule were analyzed to discover a possible strengthening effect of some of the inter-layer features, with special attention given to the so-called "I-beam" structures. These structural units are toroidal in nature which arise from 
the variation of pore diameters between layers and may act as stiffeners in a manner similar to plate girders in actual I-beams studied in civil engineering. In another recent study, Diaz et al. [25] probed the linear mechanical response of various diatom frustules to uniform compression using an experimental-simulation approach. A correlation between mechanical properties and porosity was established in that study for two diatoms, Coscinodiscus sp. and F. kerguelensis frustules via FEM, simulating flawless single-layered porous frustules with no deformation patterns.

All these recent efforts constitute a promising starting point towards a robust simulation-based analysis of the mechanical properties of diatom frustules at different length scales. However, there are still many questions without answers, including what is the structural response of diatom frustules in cases beyond uniform compression? And is there a possible relation between such structural responses and the morphogenesis of diatom frustules? In this sense, some recent work by diatom researchers, including one of the authors [26, 27], has proposed a novel way to look at diatoms: as buckled structures. In some diatom species, it is typical to find frustules naturally deformed into patterns suggestive of mechanical phenomena. One possible explanation for this apparently aberrant phenomenon is the presence of significant mechanical variables during morphogenesis in addition to the more thoroughly accounted-for chemical variables [28, 29]. If proven true, the hypothesis of mechanically-driven formation of frustules could lead to valuable insights for the development of manufacturing methods for diatom-inspired structures. In particular, the suggestion [27] that, structures with naturally developed buckled shapes, especially those in which thickening follows the appearance of such shapes, might offer increased mechanical strength, deserves further investigation. In this study, mechanics of centric diatom frustules, Coscinodiscus sp., was analyzed using FEM simulation together with independent SEM images of several characteristic deformation patterns observed in nature and 
mechanical test measurements. The difference of this approach versus previous simulations [23-25] is that the structural behaviour of diatom frustules and their response to external stimuli were investigated here at the micron scale, allowing the study of the relation between diatom morphology and mechanics in a novel and systematic fashion. The main objective was to elucidate the type of mechanical interactions that might result in these particular diatom frustules. Additionally, this study sought to improve the understanding of the relation between morphology and structural behaviour in diatom frustules.

\section{Methodology}

\subsection{SEM imaging}

The SEM micrographs used in this study were obtained from related studies [26, 27] on diatom frustules as buckled structures, a unique view on diatom morphogenesis. In those investigations, a wide range of SEM images were provided for several diatom species, with special attention given to frustule morphologies featuring specific patterns of "hills and valleys" that are suggestive of mechanical phenomena. The focus here on SEM images of circular centric diatoms is mainly due to two reasons: (i) the radial symmetry facilitates modelling, simulation and visualization of results; and (ii) most experimental data on mechanical properties of diatom frustules is available for centric species. However, the FEM methods used in this work can be easily applied to diatom frustules of any arbitrary shape. Most importantly, the main focus is on the global deformation patterns exhibited by diatom frustules naturally, not on local behaviour or features.

\subsection{Frustule model creation via CAD modelling}

Based on the independent SEM images described above, the threedimensional morphology of centric diatom frustules was modelled using the Mechanical APDL (ANSYS) CAD submodule [30]. Centric diatom frustules in nature can be approximated as two dome-shaped frustules 
(or valves) fitting together like a Petri dish and its lid. In this work, a CAD model was created to represent a single valve of each frustule by approximating the dome shape as a surface of revolution. Specifically, a revolved ellipse segment with an additional vertical rim at the end of the major axis, as shown with detail in Figure 2. The dimensions of the Coscinodiscus sp. (i.e., major and minor axes lengths and height of the vertical rim) were determined previously [11]. These overall frustule dimensions were fixed for all the calculations in this study, but the frustule thickness was varied (Table 1), as happens in nature [10, 31, 32]. An ordered array of pores was added by extruding a series of circles, following the distribution shown in the reported SEM images, and removing the associated material from the initial frustule. The resulting $3 \mathrm{D}$ porous frustule, shown in Figure 2, represents the domain on which the FEM simulations were performed. More specifically, the dimensions of each $\mathrm{CAD}$ model were based on the average dimensions obtained from SEM imaging and listed in Table 1. While the CAD model used is an approximation of the actual biological structure, it was created from SEM characterizations of diatom samples. It should be noted that the basic geometry modelled in this work is by no means a detailed reproduction of real diatom frustules. Rather it is a simplification that allows for easy and quick analysis. The pattern and geometry of pores are simplified with respect to the biological samples, and so is the frustule thickness, since the actual thickness of the frustule is not constant, particularly around the pores [11]. These approximations do not invalidate the subsequent FEM model, but indeed limit the scope of this study to the identification of "global" modes of deformation. Localized phenomena are thus beyond the scope of this work. 

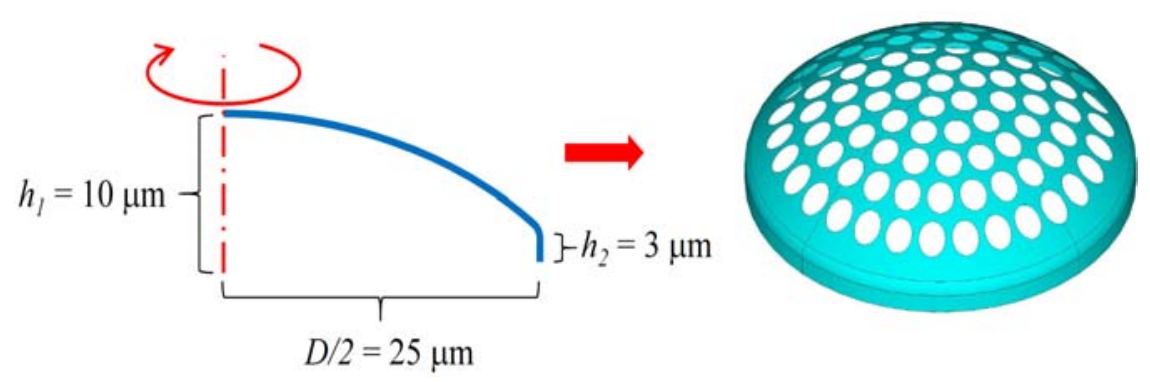

Figure 2. (Color online) CAD model of a dome-shaped diatom Coscinodiscus sp. frustule (right) with details of the generatrix curve (left). Dimensions of various frustule models are listed in Tables 1 and 2 depending on simulation.

Table 1. Results from buckling analysis of a model of Coscinodiscus $s p$. with pore diameter $d=1.7 \mu \mathrm{m}$

\begin{tabular}{|c|c|}
\hline $\begin{array}{c}\text { Frustule thickness } t \\
(\mu \mathrm{m})\end{array}$ & $\begin{array}{c}\text { Critical buckling load } P \\
(\mu \mathrm{N})\end{array}$ \\
\hline \hline 0.2 & 10.36 \\
\hline 0.4 & 77.71 \\
\hline 0.6 & 249.07 \\
\hline 0.8 & 572.45 \\
\hline 1.0 & 1099.20 \\
\hline 1.2 & 1881.90 \\
\hline 1.4 & 2971.40 \\
\hline 1.6 & 4429.30 \\
\hline 1.8 & 6307.40 \\
\hline 2.0 & 8667.20 \\
\hline
\end{tabular}


Table 2. Results from buckling analysis of a model of Coscinodiscus $s p$. with frustule thickness $t=0.4 \mu \mathrm{m}$

\begin{tabular}{|c|c|}
\hline $\begin{array}{c}\text { Pore diameter } d \\
(\mu \mathrm{m})\end{array}$ & $\begin{array}{c}\text { Critical buckling load } P \\
(\mu \mathrm{N})\end{array}$ \\
\hline \hline 1.6 & 153.32 \\
\hline 1.8 & 143.99 \\
\hline 2.0 & 134.74 \\
\hline 2.2 & 121.60 \\
\hline 2.4 & 109.03 \\
\hline 2.6 & 91.85 \\
\hline 2.8 & 75.93 \\
\hline 3.0 & 53.21 \\
\hline 3.2 & 31.93 \\
\hline
\end{tabular}

Young's modulus used in this study is within the range obtained from previous experiments [6]: $E=22.4 \mathrm{GPa}$ and $v=0.17$. Compression loads $P=10 \mu \mathrm{N}$ were applied as described in Subsection 2.3 below. Average dimensions from [11] were taken: Frustule diameter $D=50 \mu \mathrm{m}$, total height $h_{1}=10 \mu \mathrm{m}$, and vertical rim height $h_{2}=3 \mu \mathrm{m}$. See Figure 2 for more details.

\subsection{FEM simulation of centric diatom frustules}

The primary input for the FEM compression simulation of the Coscinodiscus $s p$. frustules was experimental data, specifically, Young's modulus $E$, Poisson's ratio $v$, and density $\rho$. These parameters were determined from previous experimental studies $[6,11]$ and used to define a linear computational model using the FEM module APDL Mechanical within ANSYS. The domain was discretized with shear deformable shell finite elements (SHELL63) of uniform thickness. The chosen elements had four nodes each and allowed for both membrane and bending capabilities. To ensure the mesh was appropriate to avoid any convergence errors, the diatom frustule structure was simulated multiple times under the same generic load with successive mesh refinements, monitoring the stress distribution after each simulation until stress 
convergence was reached. The resulting mesh (see Figure 3) consisted of approximately 4000 elements, with some variations in number depending on the pore size chosen for each simulation. A fixed boundary condition was imposed on the nodes along the rim to mimic the condition of electrostatic adhesion to the substrate present during experiments. A second boundary condition in which only displacements were restricted along the rim and rotations remained free (i.e., as in a 3D hinge) was also tried with no significant difference found for this kind of test. A modal analysis was first performed on the FEM model in the absence of loads to obtain the deformation modes. The results were then compared to the deformation patterns observed in SEM images of this frustule to determine whether or not these natural designs could correspond to mechanical phenomena. Additionally, the structural instability of the model was studied with a concentrated compression load $P=10 \mu \mathrm{N}$ applied vertically along the whole perimeter of the central pore.

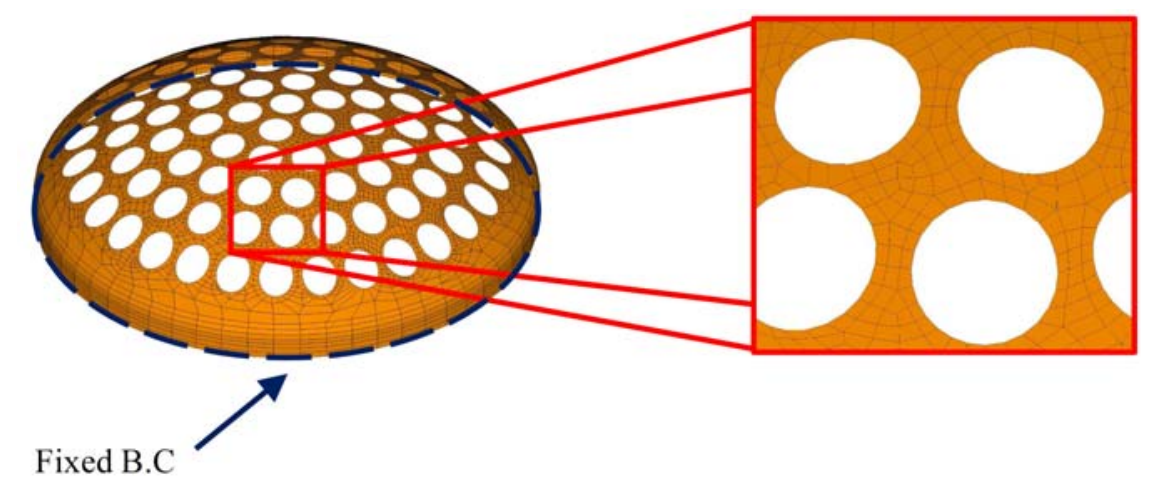

Figure 3. (Color online) Finite element discretization of a porous domeshaped Coscinodiscus sp. frustule model. Boundary conditions (B.C.) at the base are fixed to zero (both displacements $d$, and rotations $\theta$ ) and a close-up view of the mesh fitness is shown in detail. 


\subsection{Modal analysis and critical buckling load}

Two distinct types of analyses were considered in this study: modal analysis and critical buckling load. While these phenomena are treated similarly when performing simulations, they are in fact quite different. From the mechanical point of view, a diatom frustule might be subject to external mechanical loads such as those proposed by Hamm et al. [6] and deform accordingly without fracture. Alternatively, a diatom frustule might be subject to initial mechanical perturbations (impacts, sudden localized deformations, etc.) for a brief moment instead of withstanding a constant load. In this case, the mechanism in action would be closer to that of the deformation modes, which are dynamic in nature. Each of these behaviours are described next.

\subsubsection{Modal analysis}

Modal analysis consists in determining the dynamic response of a structure to a certain excitation in the absence of applied loads. It is not related to instability but instead to structural vibrations and especially to the concept of resonance. Modal analysis [33] consists of finding the solutions to the equation of motion of a structure to determine the deformation modes in which it will respond to initial conditions. In this case, a linear homogeneous material that follows Hooke's law is assumed, as shown in Equation (1):

$$
\mathbb{M} \ddot{\boldsymbol{u}}+\mathbb{K} \boldsymbol{u}=0,
$$

where $\mathbb{M}$ is the mass matrix of the structure and $\ddot{\boldsymbol{u}}$ is the second time derivative of $\boldsymbol{u}$. The general solution to this equation has the harmonic form as shown in Equation (2):

$$
\boldsymbol{u}=\varphi e^{-\tau i \omega},
$$

where $\varphi$ is a certain amplitude vector, $\tau$ is the time variable, and $\omega$ is a scalar that defines a periodic solution. If Equation (2) is substituted in Equation (1), a new eigenvalue problem is obtained: 


$$
\left[\mathbb{K}-\omega^{2} \mathbb{M}\right] \varphi=0
$$

which again gives a series of shapes, defined here by $\varphi$. In this case, each value of $\omega$ represents the specific frequency at which the structure can be excited to obtain the corresponding response. The eigenvectors $\varphi$ describe deformation shapes (deformation modes) corresponding to each particular value of $\omega$.

\subsubsection{Critical buckling load}

Buckling is a form of structural failure that occurs commonly in slender structures. This phenomenon occurs in the elastic range and in general is not dependent on the material strength. In practice, buckling failure often occurs at stress levels that are considerably below the yield stress in ductile materials or the ultimate stress in brittle ones. In practical terms, buckling can be defined as a sudden and dramatic increase in deformations for a relatively small increase in the loads. In the context of linear finite elements [33], the structural response can take the form of the system of algebraic equations (Equation (4)):

$$
\mathbb{K} \boldsymbol{u}=\boldsymbol{P},
$$

where $\mathbb{K}$ is the stiffness matrix, $\boldsymbol{u}$ is the vector containing the degrees of freedom (displacements and their derivatives), and $\boldsymbol{P}$ is the vector of applied loads. The presence of buckling implies there is more than one solution to this system of equations, which means that for a certain load increase there are more than one corresponding displacement responses. For an ideal linear elastic structure, buckling results from considering two different steps: (i) the structural stiffness matrix $\mathbb{K}$ is used to obtain a standard linear static analysis under a generic load $\boldsymbol{P}$, and (ii) the stress state obtained in this linear response is used to form the geometric stiffness matrix $\mathbb{K}_{G}$. The critical buckling loads are then calculated by solving an eigenvalue problem (Equation (5)):

$$
\left[\mathbb{K}-\lambda \mathbb{K}_{G}\right] \boldsymbol{u}=0 .
$$


Each eigenvalue $\lambda$ is a buckling load factor and represents the scalar that multiplies the applied load $\boldsymbol{P}$ to reach the buckling state. Each eigenvector $\boldsymbol{u}$ gives the displacements of that particular solution.

From all the above, the two procedures applied in this work are analogous in mathematical terms, but they represent quite different physical phenomena. In the case of the critical buckling load, the solutions obtained are particular cases of instability under the effect of the applied force, whereas in a modal analysis each solution represents a potential shape in which the structure will deform after a sudden impulse.

\section{Results}

\subsection{Modal analysis}

In this study only the first ten deformation modes were considered for analysis. This responds to two main motivations: (1) the accuracy of the numerical method used in the simulation (an iterative eigenvalue analysis) decreases for higher modes, and (2) the first ten modes usually provide enough information to describe the majority of deformation

phenomena. More elaborate cases of structural response can be interpreted as a linear combination of these ten modes. Figure 4 shows the results of the modal analysis in comparison with the deformation patterns observed via SEM. In this case, SEM micrographs of different centric diatom species, not only Coscinodiscus, were used as reference. This responds to the fact that, while the geometrical features of diatom frustules vary widely among different species, it is a well-accepted practice to group different species together by their general geometry. Namely, centric species with radial symmetry and pennate species with bilateral symmetry, as explained in Section 1. Many of the different morphologies within the realm of centric diatoms can reasonably be considered to share traits such as circular shape, thin-walled structure, and porosity. The specific deviations from this general geometry in each 
species can potentially determine localized mechanisms of deformation, but it is unlikely they will invalidate the underlying assumption of "thin shell mechanics" used in the FEM model. In this sense, it can be noted that some of the SEM images in Figure 4 show specific geometric features that have grown separate from the frustule and as such depend on each diatom species and specimen conditions. To focus the analysis, the specific global deformation of interest observed via SEM has been highlighted in each case. It can be seen that the deformation modes obtained here closely match the frustule deformation patterns obtained by SEM. It is worth noticing that some modes appear twice in the set, since there are two main axes in the plane about which the bending pattern may occur. In practice, this translates into a diatom frustule that appears "rotated" by an angle of 90 degrees about its axis. 

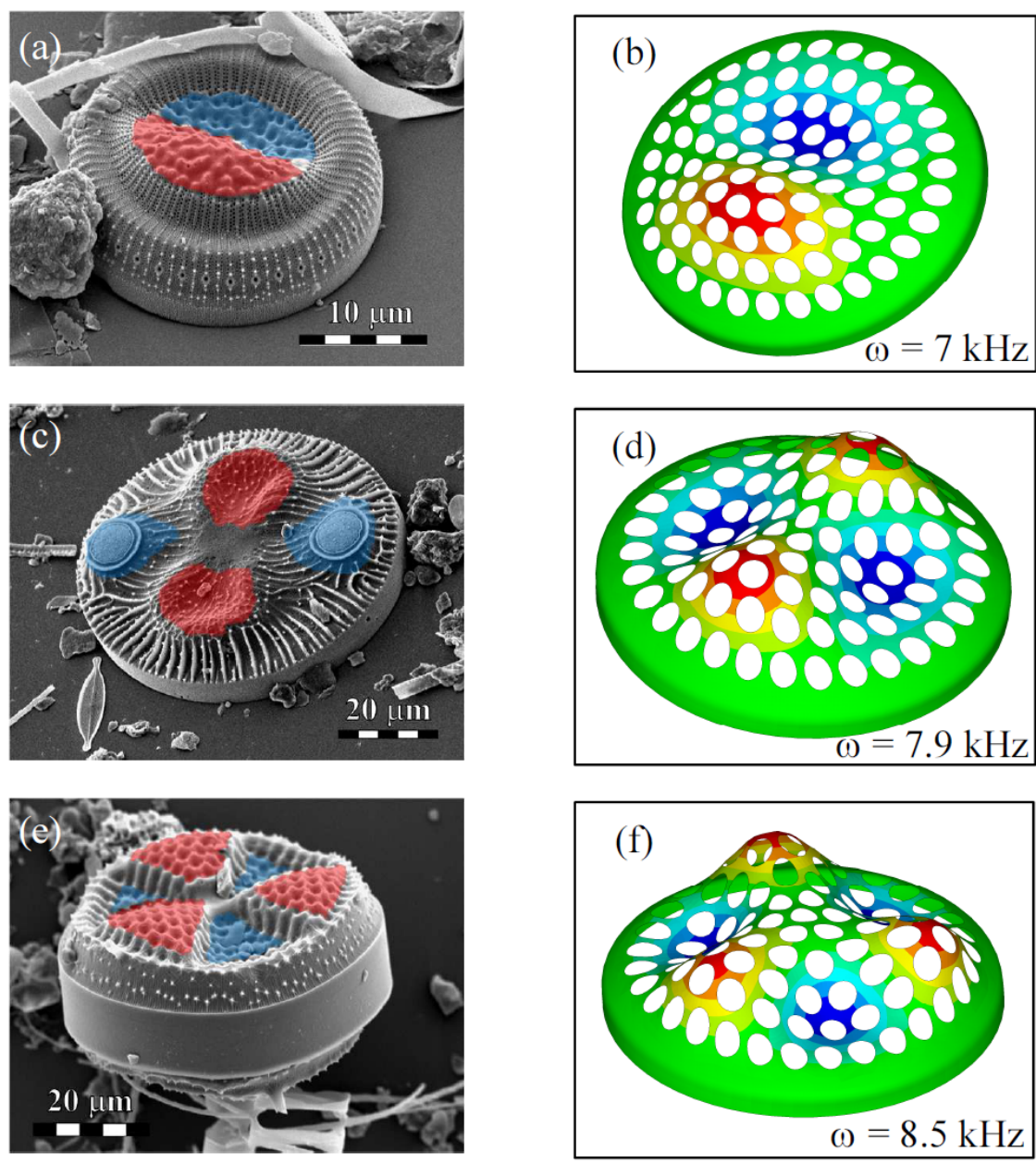

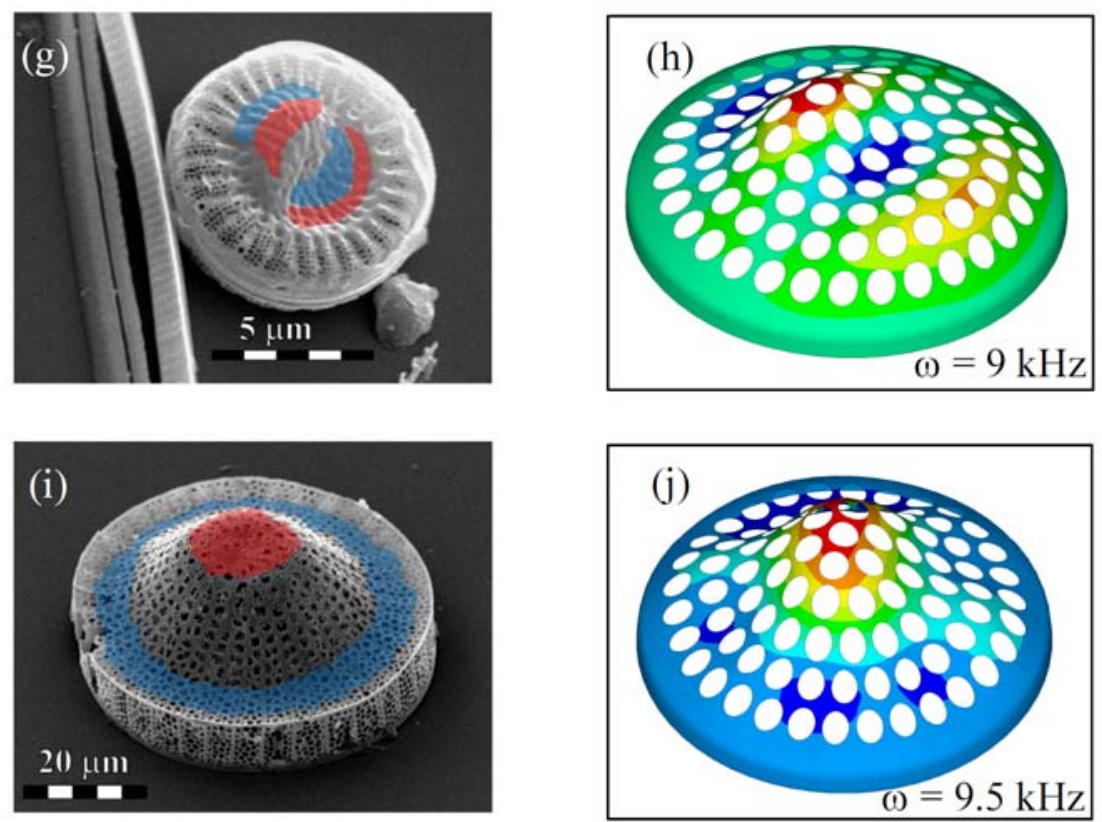

Figure 4. (Color online) Comparison of deformation patterns of centric frustules observed in experiments with their associated modal analysis (natural frequencies are shown in each case). SEM image in (a) shows a Cyclotella distinguenda hemispherical deformation pattern reminiscent of a single sine wave while (b) indicates the first deformation mode of the diatom FEM model. The cross-pattern shown for Auliscus sculptus in (c) matches the second deformation mode of the structure shown in (d). A triangular pattern is observed in Actinoptychus senarius (e) and its FEM counterpart is the third deformation mode in (f). A double-wave hemispherical pattern is present in Cyclotella distinguenda (g) which corresponds to the fourth mode shown in (h). The radial pattern in Coscinodiscus asteromphalus (i) matches the simulated fifth mode shown in (j). The original SEM images of centric frustules [27] have been intentionally color-shaded to better show the deformation patterns. Scale bars were added on the SEM images with permission of their authors. 


\subsection{Effect of morphology on structural instability}

Next, the FEM linear buckling analysis of a centric diatom frustule under a concentrated compressive load applied at the center of the dome was performed. In this case, no attempt was made to correlate the buckling modes with the experimentally observed deformation patterns. This is due to concentrated loads of this type being very unlikely to be present in the normal environment of diatoms. Instead, the same simulation was performed repeatedly for different frustule geometries to establish the relation between morphology and the onset of buckling. Specifically, frustule thickness and pore diameter were systematically examined.

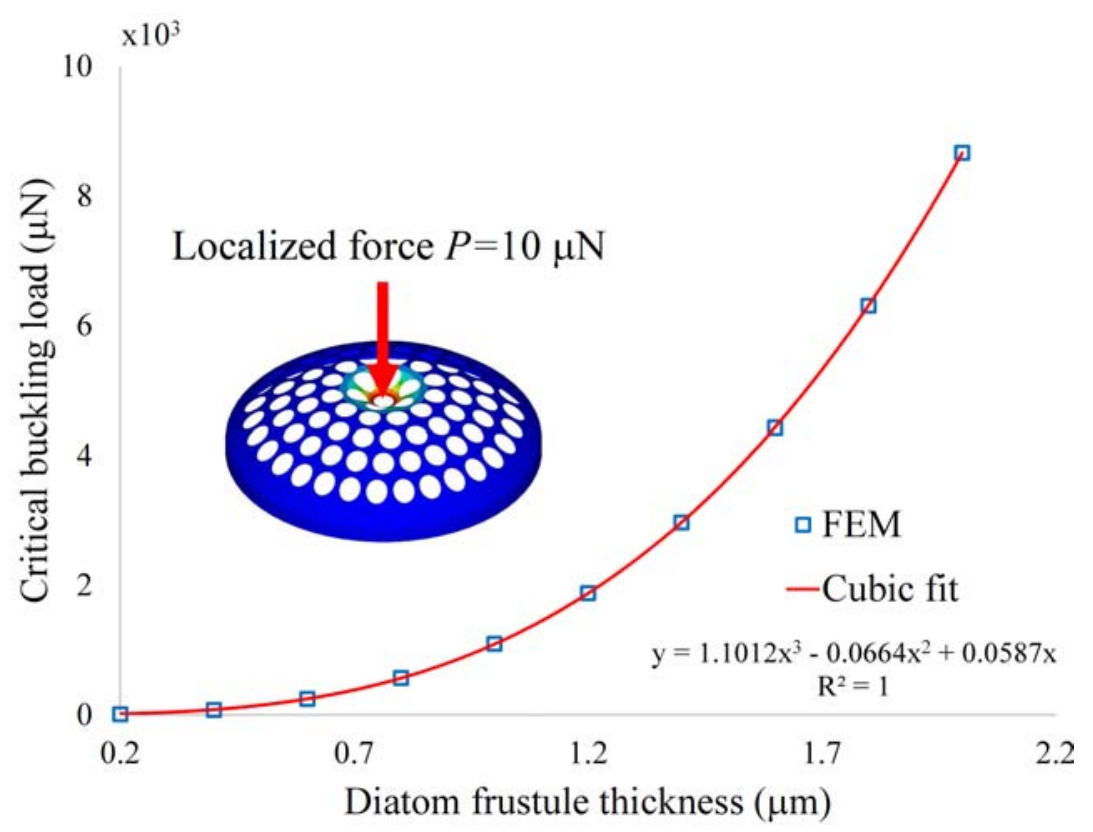

Figure 5. (Color online) Effect of morphology on the structural response of a centric frustule. A cubic relation is observed between the frustule thickness and critical buckling load. Frustule diameter $D=50 \mu \mathrm{m}$. Pore diameter $d=1.7 \mu \mathrm{m}$. See Table 1 for additional data. 
Figure 5 depicts the critical buckling loads of a centric diatom frustule for different values of thickness. The simulation was performed for a range of frustule thickness from 0.2 to 2 microns (Table 1), with the intent of covering all possible natural geometries. The resulting correlation was successfully approximated with a cubic polynomial constrained to go through the origin. In addition, the same concentrated load was applied to frustules with different pore sizes as shown in Table 2 (while keeping the original frustule thickness $t=0.4 \mu \mathrm{m}$ ) and a distinct correlation was observed. Figure 6 shows these results, to which a distinct quadratic fit was successfully applied. It should be noted that the simulation was performed for a wide range of pore sizes (i.e., pore diameters) while keeping the same pore distribution (i.e., number of pores and position of pores over the frustule were kept constant) in order to isolate the effect of a single variable (pore size) on the frustule stability.

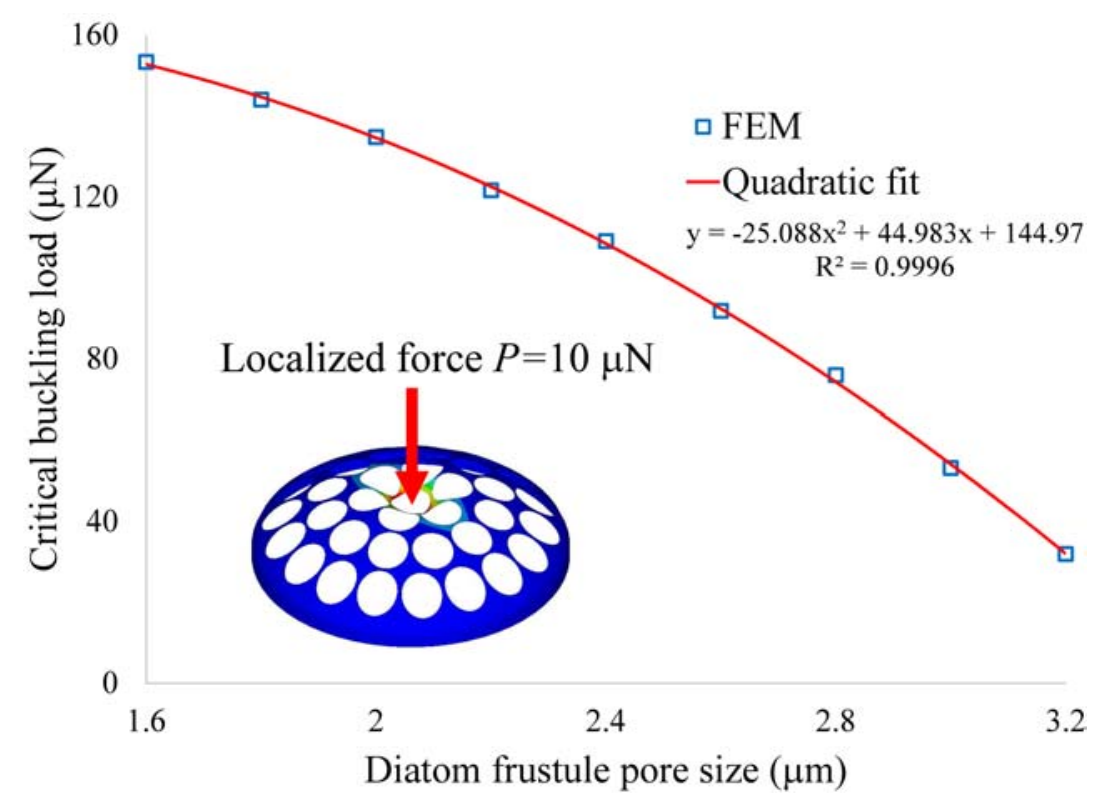

Figure 6. (Color online) Effect of morphology on the structural response of a similar centric frustule (thickness $t=0.4 \mu \mathrm{m}$, diameter $D=50 \mu \mathrm{m}$ ), indicating an inverse quadratic relation is observed between the frustule pore size and critical buckling load. See Table 2 for associated information. 


\section{Discussion}

Coscinodiscus $s p$. frustules have been well characterized via SEM prior to any simulations in this study in terms of frustule morphology and porous structure. A wide range of SEM micrographs with naturally deformed centric diatom frustules were first selected, which were deemed suitable representatives used for further analyses. The objective of this approach was to ensure any subsequent comparisons were made to the global deformation trends commonly observed in many centric frustules and not in localized specific features that are not observed generally and that cannot be readily reproduced with the simplified FEM models used in this work. SEM images of the frustules were used for creation of 3D diatom models, with average experiment-based dimensions in morphological variables such as frustule diameter, pore size, and frustule thickness. Using FEM, the mechanics of the centric frustules was then investigated. A full and highly precise 3D model would be useful to provide additional information in terms of local stress distributions and deformations, but it is not expected to affect the global deformation patterns presented in this study.

FEM simulation as a method of analysis is common in the mechanical study of structures, although it has seldom been used in the case of diatom mechanics. Given that the analyses performed in this work were linear, the results should be taken only as a first approximation to the problem. Further studies could make use of nonlinear mechanics to analyze plastic deformations and/or fracture in diatom frustules. In addition, measures have been taken in the present study to minimize numerical errors and provide the most accurate approximation possible under linear conditions. A considerably refined mesh has been used to minimize approximation errors and the boundary conditions have been specifically chosen to correspond to the experimental case (nanomechanical test) in which the diatom frustule is electrostatically adhered to a hard substrate such as a Si wafer. 
The resemblance between the FEM results and SEM images in Figure 4 leads to the idea of diatom frustules being subject to mechanical interactions. The deformation modes obtained from simulation provide possible "free" responses of a diatom frustule to an initial dynamic perturbation. In this sense, the word "free" refers to an absence of resistance to outside forces. In practical terms, the dynamic response of a structure to an initial perturbation is a linear combination of all the deformation modes, with different modes having different weights depending on the specific conditions of the initial stimulus. The myriad of deformation patterns observed experimentally could mean the deformations result from the same basic phenomenon, but differ in the initial conditions. Furthermore, while most interactions in aqueous environments in which diatoms grow and live indeed occur at low values of Reynold's number [11], dynamic perturbations are entirely possible even at the micro and nanoscale of diatoms. This represents a possible mechanism in which subtle internal forces, perhaps generated by the cytoskeleton at the right moment during morphogenesis, produce specific structural responses that remain visible as the frustule thickens. For example, when the microfilament ring [7, 34] at the perimeter of the silicalemma turns the corner at the vertical rim (see Figure 2 for the geometry), it may suddenly cease to be in mechanical opposition to the microtubules attached to the surface of the silicalemma and extending radially from the center of the forming frustule [9] (see Figure 4).

As shown in Figure 5, the critical buckling load of a diatom frustule increases monotonically with frustule thickness, which means any mechanical buckling phenomenon is more likely to occur during the early stages of diatom morphogenesis. The cubic fit applied in this figure responds to the standard theory of plates [35], in which the flexural stiffness is proportional to $t^{3}$. One avenue towards elucidating other possible buckling mechanics would be switching the focus from external (e.g., predatorial) mechanical loads to consider instead the response of immature frustules to internal loads acting during diatom morphogenesis [34]. In this sense, the mechanics of growth of the silicalemma [7] (the membrane on which the diatom valve is formed during morphogenesis) 
and the possible constraints imposed by the diatom frustule must be studied with more detail to determine planes and directions in which mechanical loads are applied. Most importantly, it is at this stage of growth that the possibility of buckling is greater, since immature diatom frustules are thinner and less rigid than their fully formed counterparts.

Further examination of Figures 5 and 6 suggests the correlation between morphology (pore size and frustule thickness) with deformation modes is indeed significant to the strength of diatom frustules. This relation could serve as a starting point for a design methodology of diatom-inspired structures based on careful selection of morphology parameters. A quadratic fit was applied in Figure 6 to describe the relation between stiffness and pore size as closely as possible. The results could also set the ground for studies on the role of morphology during diatom morphogenesis and the onset of instability phenomena. Further studies on this respect could focus on the effects of different pore shapes and sizes and how these changes in volume distribution account for changes in mechanical behaviour.

Frustule thickness and pore size were chosen as variables in the morphology section of this study for two reasons: (1) they allow researchers to easily categorize diatoms of different species based on geometric criteria and (2) when attempting to design diatom-inspired microdevices for optimal mechanical performance, parametrization of the morphology will be essential. In this sense, pore size and frustule thickness are natural candidates for the main parameters defining diatom morphology. Future studies in this respect will be based on adaptive methods to select the best combination of these variables to achieve the desired results.

This study has focused on mainly natural deformation modes instead of bifurcation and buckling modes. While this may also be a relevant phenomenon, the difficulty in establishing the proper boundary conditions and loading cases for the frustule limits its possible inclusion in this study. A more detailed, fully three-dimensional frustule model would be needed. 
Additionally, the presence of cracks or other initial imperfections in the frustule was not considered in this study. It is expected these elements would reduce the critical buckling load for a given frustule. If cracks were localized and sufficiently large, they would also determine the specific buckling mode that would occur.

The results presented in this work depend significantly on the choices made during the creation of the diatom CAD model. Even though the dimensions and shape were carefully chosen to reproduce the average diatom frustule, there is significant room for variation even within a single diatom species. One of the most significant elements to take into account in this case is the curvature of the dome-shaped valve. The dome shape was approximated as the revolution of an ellipse segment whose axes were the average diameter and height of the Coscinodiscus $s p$. valves. Different curvatures could be used instead of this, or the frustule could be considered flat-top [25]. However, it is unlikely such changes would significantly alter the deformation modes of the structure, which closely resemble the Bessel function solution to the vibrations of a circular membrane [27, 36]. More significant insights would be obtained by analyzing pennate shapes instead, since the circular symmetry would be lost. In this case, it is reasonable to expect global bending patterns reminiscent of Euler's buckling shapes [37], since the elongated geometry would be similar to a thin-walled column.

Another potential area of study is the hierarchical arrangement of layers observed in some species of diatoms. In the case of Coscinodiscus $s p$., previous studies [11] have shown the presence of toroidal ridges between the bottom and mid layers. These ridges resemble I-beam structures in cross-section and could potentially have a stiffening role in the mechanics of diatom frustules. To determine the role of these features, a fully 3D FEM model (brick elements instead of shell elements) would be needed. Similarly, future studies could focus on local effects by modelling the mechanics of the basic arrangement of pores that repeats 
periodically over the frustule. This basic component could be modelled as a hexagonal array of pores or other representative geometry to determine stress distributions over the pores and the contributions of each layer to overall mechanical properties.

\section{Conclusions}

In summary, methodic studies of the mechanics of centric frustules revealed some correlations between their deformation patterns and diatom morphology. Specifically, FEM modal analysis indicates that deformation modes correlate well with SEM deformation patterns. The implications of these findings reported in this study are potentially farreaching. These include the ability to design optimum morphologies (using maximum and minimum values) of new diatom-inspired structures, investigate the effect of hierarchical layers considering buckling conditions, and map strategies for the fabrication of nanostructures and nanodevices.

Future experiments and simulation studies will need to include complete diatom frustule morphology (full valve and hierarchical structure) as well as other diatom species to more completely identify most appropriate design criteria for nanotechnology applications.

Centric diatoms vary in at least three basic parameters: diameter of the valve, thickness of the valve, and diameter of the pores. Due to the critical buckling load, future studies could involve predictions that centric frustules evolve so that these three parameters cannot have arbitrary values, but rather are set so that typical forces in the environment (turbulence, predation, etc.) do not exceed the critical buckling load. If individual diatoms were plotted in this threedimensional space, there should therefore be regions of this "morphospace" without any representative diatoms. In an analogous tally of snail shells, regions with and without representative species were set apart [38]. 
If forming diatom frustules buckle first and then thicken, and if the buckled shapes prove mechanically stronger than their unbuckled counterparts, then a basis for a new form of nanoconstruction of strong materials may arise. Perhaps this approach is even scalable to buildings and other large human-made structures. In this sense, the findings in this paper constitute a first step towards fabrication techniques based on the creation of the "hills and valleys" deformation patterns observed in Subsection 3.1 by means of dynamic micromanipulation of diatominspired structures. This basic idea could potentially be extended to other geometries by pursuing a parametric study of the relation between morphology and mechanical properties at the microscale. Such a generalization would represent a major step forward in the development of new materials with tailored properties.

Given the eigenvectors resulting from the modal analysis, the authors predict that in air a diatom frustule would exhibit mechanical resonance frequencies in the range of $7-10 \mathrm{KHz}$. Those same frustules have light focusing properties [39]. Therefore, it may be possible to combine both properties to make novel optomechanical devices.

The findings presented in this study contribute toward understanding porous bio-structures such as diatoms and developing advanced materials for a wide range of applications by tailoring their morphologies and structures. Further progress in materials innovation, experimental, and simulation methods as well as additive manufacturing will greatly aid current efforts to fully characterize the effect of microstructures on associated properties, and to design for performance along multiple length scales. Recent development of high-resolution computerized tomography [40] indicates that studies such as the presented work here are needed for the faster fabrication of 3D complex materials with sub-micron resolution. Additionally, further investigations regarding the mechanical properties of diatoms under varied environmental conditions may be inspired. 
Finally, the key advances of this work are:

- An experiment-based simulation approach has been introduced to model the mechanics of diatom frustules. This approach is simple and easy to use and at the same time is accurate enough to reproduce the global deformation patterns observed in centric frustules via SEM.

- There is a clear correspondence between the deformation patterns commonly observed in centric diatom frustules and the deformation modes of thin, porous dome with dimensions and material properties corresponding to diatom frustules. This suggests the observed deformation patterns may be the result of mechanical interactions during morphogenesis.

- The study of diatoms using thin shell mechanics opens the door to new possibilities in the study of diatom-inspired devices based on the unique mechanical properties of diatom frustules and taking advantage of their specific response to mechanical manipulation for design and fabrication purposes.

\section{Acknowledgements}

The authors would like to thank Mary Ann Tiffany (San Diego State University, retired) for sharing her high-resolution SEM micrographs for analysis and Mike Dunlap (University of California, Merced) for technical assistance. This work was performed under the auspices of an internal grant, which supported co-author (AG). In addition, a research award from the Academic Senate Committee on Research at the University of California, Merced provided funds for research supplies and conference travel expenses. Dedicated to the memory of Luis Davila Negron, leaving a vibrant legacy of kindness and authenticity (father of co-author LD) and Frithjof Sterrenburg, inspiring diatomist par excellence [14]. 


\section{References}

[1] G. Fleming and M. Ratner, Grand challenges in basic energy sciences, Physics Today 61(7) (2008), 28.

[2] U. S. N. N. Initiative, Nanotechnology 101, [Online], [Accessed 21 April 2017].

Available: http://www.nano.gov/nanotech-101/special

[3] P.-Y. Chen, J. McKittrick and M. Meyers, Biological materials: Functional adaptations and bioinspired designs, Progress in Materials Science 57(8) (2012), 1492-1704.

[4] S. Weiner and L. Addadi, At the cutting edge, Science 298(5592) (2002), 375-376.

[5] N. Kröger and E. Brunner, Complex-shaped microbial biominerals for nanotechnology, WIREs Nanomedicine and Nanobiotechnology 6(6) (2014), 615-627.

[6] C. Hamm, R. Merkel, O. Springer, P. Jurkojc, C. Maier, K. Prechtel and V. Smetacek, Architecture and material properties of diatom shells provide effective mechanical protection, Nature 421 (2003), 841-843.

[7] F. Round, R. Crawford and D. Mann, The Diatoms, Biology \& Morphology of the Genera, Cambridge: Cambridge University Press, 1990.

[8] D. Mann and S. Droop, Biodiversity, biogeography and conservation of diatoms, Hydrobiologia 336(1-3) (1996), 19-32.

[9] R. Gordon, D. Losic, M. Tiffany, S. Nagy and F. Sterrenburg, The glass menagerie: Diatoms for novel applications in nanotechnology, Trends in Biotechnology 27(2) (2009), 116-127.

[10] T. Fuhrmann, S. Landwehr, M. El Rharbi-Kucki and M. Sumper, Diatoms as living photonic crystals, Applied Physics B Lasers and Optics 78(3-4) (2004), 257-260.

[11] D. Losic, G. Rosengarten, J. G. Mitchell and N. H. Voelcker, Pore architecture of diatom frustules: Potential nanostructured membranes for molecular and particle separations, Journal of Nanoscience and Nanotechnology 6(4) (2006), 1-8.

[12] M. Bariana, M. Sinn Aw and D. Losic, Tailoring morphological and interfacial properties of diatom silica microparticles for drug delivery applications, Advanced Powder Technology 24(4) (2013), 757-763.

[13] H. Aldersey-Williams, Towards biomimetic architecture, Nature Materials 3(5) (2004), 277-279.

[14] F. Sterrenburg, Crystal palaces - diatoms for engineers, Journal of Nanoscience and Nanotechnology 5(1) (2005), 100-107.

[15] L. Willis, K. Page, D. Broomhead and E. Cox, Discrete free-boundary reactiondiffusion model of diatom pore occlusions, Plant Ecology and Evolution 143(3) (2010), 297-306.

[16] L. Medlin, The use of the terms centric and pennate [+erratum: 25(2), 461], Diatom Research 24(2) (2009), 499-501. 
[17] C. Hamm, The evolution of advanced mechanical defenses and potential technological applications of diatom shells, Journal of Nanoscience and Nanotechnology 5(1) (2005), 108-119.

[18] N. Almqvist, Y. Delamo, B. L. Smith, N. H. Thomson, Å. Bartholdson, R. Lal, M. Brzezinski and P. Hansma, Micromechanical and structural properties of a pennate diatom investigated by atomic force microscopy, Journal of Microscopy 202(3) (2001), 518-532.

[19] G. Subhash, S. Yao, B. Bellinger and M. Gretz, Investigation of mechanical properties of diatom frustules using nanoindentation, Journal of Nanoscience and Nanotechnology 5(1) (2005), 50-56.

[20] D. Losic, K. Short, J. Mitchell, R. Lal and N. Voelcker, AFM nanoindentations of diatom biosilica surfaces, Langmuir 23(9) (2007), 5014-5021.

[21] M. Buehler and H. Gao, Atomistic studies of flaw tolerant nanoscale structural links in biological materials, Nanomechanics of Materials and Structures, Netherlands: Springer (2006), 139-150.

[22] S. Keten, Z. Xu, B. Ihle and M. Buehler, Nanoconfinement controls stiffness, strength and mechanical toughness of beta-sheet crystals in silk, Nature Materials 9(4) (2010), 359-367.

[23] A. García, D. Sen and M. Buehler, Hierarchical silica nanostructures inspired by diatom algae yield superior deformability, toughness, and strength, Metallurgical and Materials Transactions A 42(13) (2011), 3889-3897.

[24] J. Lu, C. Sun and J. Wang, Mechanical simulation of a diatom frustule structure, Journal of Bionic Engineering 12(1) (2015), 98-108.

[25] M. Diaz, K. Ma, J. Schoenung and L. Dávila, An integrated approach for probing the structure and mechanical properties of diatoms: Toward engineered nanotemplates, Acta Biomaterialia 25 (2015), 313-324.

[26] M. A. Tiffany, S. Nagy, N. Rattanawangcharoen and R. Gordon, The buckling of diatom valves, in North American Diatom Symposium (NADS), Milford, 2009.

[27] R. Gordon and M. A. Tiffany, Possible buckling phenomena in diatom morphogenesis, in The Diatom World, Springer (2011), 245-271.

[28] R. Gordon and R. W. Drum, The chemical basis of diatom morphogenesis, International Review of Cytology 150(421-22) (1994), 243-372.

[29] E. Brunner, C. Gröger, K. Lutz, P. Richthammer, K. Spinde, and M. Sumper, Analytical studies of silica biomineralization: Towards an understanding of silica processing by diatoms, Applied Microbiology and Biotechnology 84(4) (2009), 607-616.

[30] E. Madenci and I. Guven, The Finite Element Method and Applications in Engineering using ANSYS, New York: Springer, 2006.

[31] L. Friedrichs, A simple cleaning and fluorescent staining protocol for recent and fossil diatom frustules, Diatom Research 28(3) (2013), 317-327. 
[32] E. Paasche, Silicon content of five marine plankton diatom species measured with a rapid filter method, Limnology and Oceanography 25(3) (1980), 474-480.

[33] O. Zienkiewicz, R. L. Taylor and J. Z. Zhu, The Finite Element Method its Basis and Fundamentals, London: Butterworth-Heinemann, 2005.

[34] B. Tesson and M. Hildebrand, Extensive and intimate association of the cytoskeleton with forming silica in diatoms: Control over patterning on the meso- and micro-scale, PLoS One 5(12) (2010), 1-13.

[35] A. Nettles, Basic mechanics of laminated composite plates, Marshall Space Flight Center, AL: National Aeronautics and Space Administration (NASA), 1994.

[36] A. Leissa, Vibrations of Plates, Washington D.C.: National Aeronautics and Space Administration, 1969.

[37] T. Galambos and A. Surovek, Structural Stability of Steel: Concepts and Applications for Structural Engineers, Hoboken: Wiley, 2008.

[38] D. Raup, Geometric analysis of shell couling: General problems, Journal of Paleontology 40(5) (1966), 1178-1190.

[39] M. M. Ghobara, N. Mazunder, V. Vinayak, I. C. Gebeshuber, M. A. Tiffany, B. Schoefs and R. Gordon, On light and diatoms: A photonics and photobiology review, In: Diatoms: Fundamentals and Applications (Submitted, 2017).

[40] R. Wagner, J. Jungck and D. V. Loo, Sub-micrometer X-ray tomography of radiolarians: Computer modeling and skeletonization, Microscopy Today 23(5) (2015), 18-23. 